\title{
Was ist eine gute Pharmacovigilance-Meldung?
}

\author{
EIViS kommt - im Oktober dieses Jahres wird Swissmedic das Elektronische \\ Vigilance System zur Online-Eingabe vermuteter unerwünschter Arzneimittel- \\ wirkungen (UAW) einführen. Nachfolgend informieren wir Sie, welche Meldungen \\ für die Arzneimittelsicherheit besonders wichtig sind.
}

Rudolf Stoller ${ }^{a}$,

Karoline Mathys ${ }^{b}$,

Martina Schäublinc,

Christoph Küngd

a Senior Expert, Abt. Arzneimittelsicherheit, Swissmedic

b Dr. pharm., Leiterin Bereich Marktüberwachung, Swissmedic

c Dr. med., Leiterin Einheit Vigilance, Abt. Arzneimittelsicherheit, Swissmedic

d Dr. phil. II, Leiter Abteilung Arzneimittelsicherheit, Swissmedic
Korrespondenz:

Dr. med. Martina Schäublin Swissmedic, Schweizerisches Heilmittelinstitut Hallerstrasse 7 Postfach

CH-3000 Bern 9

Tel. 0584638623

martina.schaeublin[at] swissmedic.ch
Ab kommenden Oktober soll das neue System ElViS den Fachleuten die Online-Meldung unerwünschter Arzneimittelwirkungen ermöglichen. ElViS wird eine direkte Verbindung zwischen Fachleuten und regionalen Pharmacovigilance-Zentren (RPVZ) herstellen und auch den Informationsaustausch mit Swissmedic verbessern. Vertreter von FMH, PharmaSuisse und GSASA, der Gesellschaft Schweizerischer Amts- und Spitalapotheker sowie die RPVZ wurden und werden bei der Entwicklung und Testung einbezogen. Ärztinnen und Ärzte sowie Apotheker/innen werden bei der Einführung des Systems über das Vorgehen orientiert. ElViS wird weitgehend selbsterklärend sein und bei Bedarf Unterstützung bei der Eingabe bieten. Aktuell ruft Swissmedic in Erinnerung, welche UAW-Meldungen für die Arzneimittelsicherheit besonders wichtig sind und was bei der Eingabe zu beachten ist. Entscheidend ist eine fokussierte Beschreibung der UAW. Für eine umfassendere Darstellung verweisen wir auf die Publikation in der SÄZAusgabe 4/2013 [1].

Die Spontanerfassung - in der Schweiz und international, besonders im Verbund des «WHO Programme for International Drug Monitoring» - ist weiterhin das entscheidende Instrument, um Sicherheitssignale bei Medikamenten auf dem Markt rasch zu erkennen und den Risiken dann zu begegnen. Sie basiert auf der Mitarbeit und dem Verständnis der Fachleute, die im Sinne der «Good Pharmacovigilance Practice» die «richtige Meldung in der richtigen Qualität zur richtigen Zeit» übermitteln. Wichtig ist, das Ungewöhnliche der UAW aufzuzeigen sowie schwerwiegende UAW zu melden.

\section{Das Ziel: frühzeitig Sicherheitssignale erken-} nen und risikomindernde Massnahmen treffen Die Spontanerfassung hat zum Ziel, mehr über bekannte Arzneimittelrisiken zu lernen und neue, bisher unbekannte unerwünschte Wirkungen zu erkennen.

Ein Sicherheitssignal ist entsprechend definiert als

1. ein vermuteter neuer Aspekt eines bekannten Risikos (der Regelfall in der Pharmacovigilance) oder

2. ein vermutetes neues, bisher unbekanntes Risiko.
Spontanmeldungen allein sind kaum je beweisend für eine UAW. Ein Signal, das aus ihnen resultiert, ist primär eine Vermutung, die weiter abgeklärt werden muss. Wird es bestätigt, erfordert es risikomindernde Massnahmen. Je höher das Gefährdungspotential z. B. schwer oder spät erkennbares Problem, schwerwiegende Schädigung des Patienten, hohe Zahl der Exponierten -, desto früher sind Massnahmen einzuleiten und umso eingreifender sind sie.

Als Beispiel für ein ebenso bekanntes wie bedeutendes Risiko seien die Blutungen unter den oralen Antikoagulantien erwähnt; die Spontanerfassung in der Schweiz und international verfolgt dazu laufend wichtige Aspekte. Aktuelle Signale bei den neuen wie den herkömmlichen oralen Gerinnungshemmern betreffen z. B. die pharmakodynamische Interaktion mit anderen Medikamenten wie Heparin, Aspirin, nicht-steroidalen Entzündungshemmern, Antidepressiva vom Typ der SSRI, pharmakokinetische Wechselwirkungen (über CYP-Enzyme, Transporter wie OATP oder den Einfluss der Nierenfunktion). Zusätzliche Probleme bei den neuen oralen Antikoagulantien entstehen durch das Fehlen eines etablierten Antidots bei Überdosierung oder akuter Blutung; aktuelle Meldungen betreffen auch das Vorgehen beim Wechsel auf einen anderen Gerinnungshemmer und nicht zuletzt das therapeutische Monitoring. Mit der alleinigen Meldung der Blutungskomplikation ist es somit nicht getan, sondern es gilt, die neuen Aspekte resp. Sicherheitssignale zu dokumentieren. Swissmedic publiziert ihre Analyse der UAWMeldungen und orientiert über die Vorsichtsmassnahmen [2]. Fach- und Patienteninformation werden regelmässig überprüft und entsprechend aufdatiert.

Als Beispiele für die zweite Kategorie - neue und überraschend auftretende UAW - sei das Auftreten von Narkolepsie nach Impfung gegen die pandemische Grippe H1N1 2009 mit Pandemrix ${ }^{\circledast}$, einem adjuvantierten Pandemie-Impfstoff, aufgeführt. Die Betroffenen erkrankten erst Monate nach der Impfung. Das Risiko wurde nur dank hoher Aufmerksamkeit der Meldenden anhand einer Häufung von Berichten zunächst in skandinavischen Ländern erkannt. In der Schweiz wurden bisher insgesamt 
Abbildung 1

Die Organisation der Pharmacovigilance in der Schweiz ist unverändert.

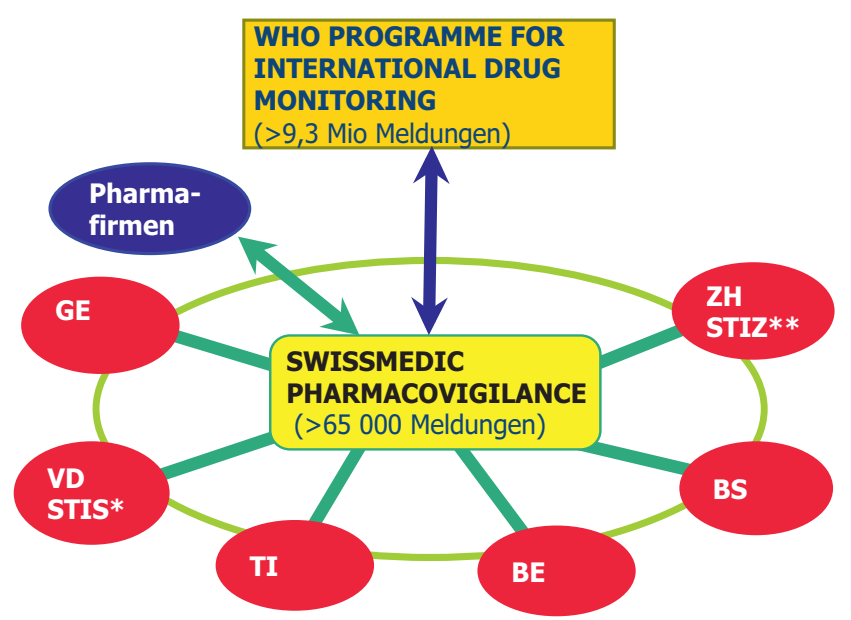

* STIS: Swiss Teratogen Information Service (Medikamentenrisiken in Schwangerschaft und Stillzeit) ** STIZ Schweiz. Toxikologisches Informationszentrum, Zürich (Intoxikationen)

9 Erkrankungen nach Injektion eines PandemieImpfstoffs berichtet. Ausgedehnte epidemiologische Studien deuten inzwischen auf einen Zusammenhang zwischen den Narkolepsie-Erkrankungen und dem Impfstoff Pandemrix ${ }^{\circledast}$ hin. Dieser wurde nur während der Grippe-Pandemie verwendet und ist nicht mehr auf dem Markt.

\section{Die gute UAW-Meldung}

Die Signale charakterisiert:

Es ist etwas Unerwartetes, Überraschendes geschehen. Im ersten Fall geht es um in der Arzneimittelinformation mehr oder weniger deutlich erwähnte wichtige Probleme bei einer bekannten UAW, die aber beim Gebrauch von Medikamenten im Alltag schwer zu berücksichtigen sind oder für welche es noch keine etablierte Lösung gibt. Im zweiten ist die UAW an sich neu und ungewöhnlich.

- Es geht um relevante, potentiell schwerwiegende Risiken.

Es sind somit diese ungewöhnlichen oder ungewöhnlich schwerwiegenden Situationen, Aspekte, Erfahrungen mit UAW, die eine Meldung auslösen und in der Beschreibung besonders hervorgehoben werden sollten. Das Online-System wird diese im Wortlaut in die Datenbank übertragen. Die Meldung sollte die wichtigen Charakteristika des Ereignisses enthalten, namentlich in Bezug auf

- Intensität und Schweregrad der UAW (Beispiel: Hepatitis mit Ikterus, wenn die Arzneimittelinformation nur erhöhte Transaminasen aufführt),

- Chronologie (Beispiel: Leberschädigungen bis zu 6 Wochen nach Absetzen unter Co-Amoxyclav, Flucloxacillin oder verwandten Substanzen),
- prädisponierende Faktoren (Beispiel: Niereninsuffizienz - Lithiumintoxikation),

- Probleme beim Gebrauch (Beispiel: tägliche statt wöchentliche Einnahme von Low-Dose Methotrexat in der rheumatologischen oder dermatologischen Indikation, mit der Folge schwerer akzidenteller Intoxikationen).

Zum konkreten Vorgehen siehe Kasten.

\section{Die Organisation der Pharmacovigilance in der Schweiz ist unverändert}

Die Meldungen gehen auch nach Einführung des Online-Systems an die regionalen Pharmacovigilance-Zentren. Wie bisher werden sie, in vollständig anonymisierter Form was Patienten, Melder, beteiligte Fachleute und Institutionen betrifft, in der Swissmedic Datenbank und der internationalen

\section{UAW melden: Was, wann und wie?}

Die Meldepflicht der Fachleute ist in Art. 59 des Heilmittelgesetzes HMG festgelegt.

\section{Welche UAW bis wann melden?}

Schwerwiegende UAW - innerhalb von 15 Tagen:

- tödlich oder lebensbedrohend

- bleibender Schaden

- Hospitalisation / verlängerte Hospitalisation

- «medizinisch wichtige» UAW (Beispiele Coupierung eines epileptischen Anfalls oder einer Hypoglykämie in der Praxis, so dass schwerere Folgen ausbleiben; Impfstoff-Versager)

Neue UAW - falls nicht schwerwiegend - innerhalb von 60 Tagen

\section{Wie melden?}

Strukturierte Eingabe gemäss Formular (künftig bevorzugt online)

- Angaben zum Patienten, Datum, Schweregrad der UAW

- ursächlich vermutete Medikamente mit Dosierung und Indikation

- weitere Medikamente

- prädisponierende Faktoren (z. B. Nierenfunktion - Kreatinin oder Kreatininclearance vor der UAW)

Beschreibung der UAW (Freitext)

- Diagnose und Symptome / relevante Befunde

- hervorgehoben die überraschenden Aspekte der UAW

- Verlauf der UAW in Bezug zu den Medikamenten (Zeit bis Auftreten, bis Besserung nach Absetzen - welches Medikaments / welcher Medikamente?)

- Differentialdiagnose - nicht-medikamentöse Ursachen (bei Leberschädigungen z. B. Alkoholkonsum, Obstruktion der Gallenwege, Angaben zu Virusserologien)

- Probleme bei Verordnung, Abgabe oder Gebrauch 
Abbildung 2

Die Zahl der Meldungen steigt weiterhin deutlich an.

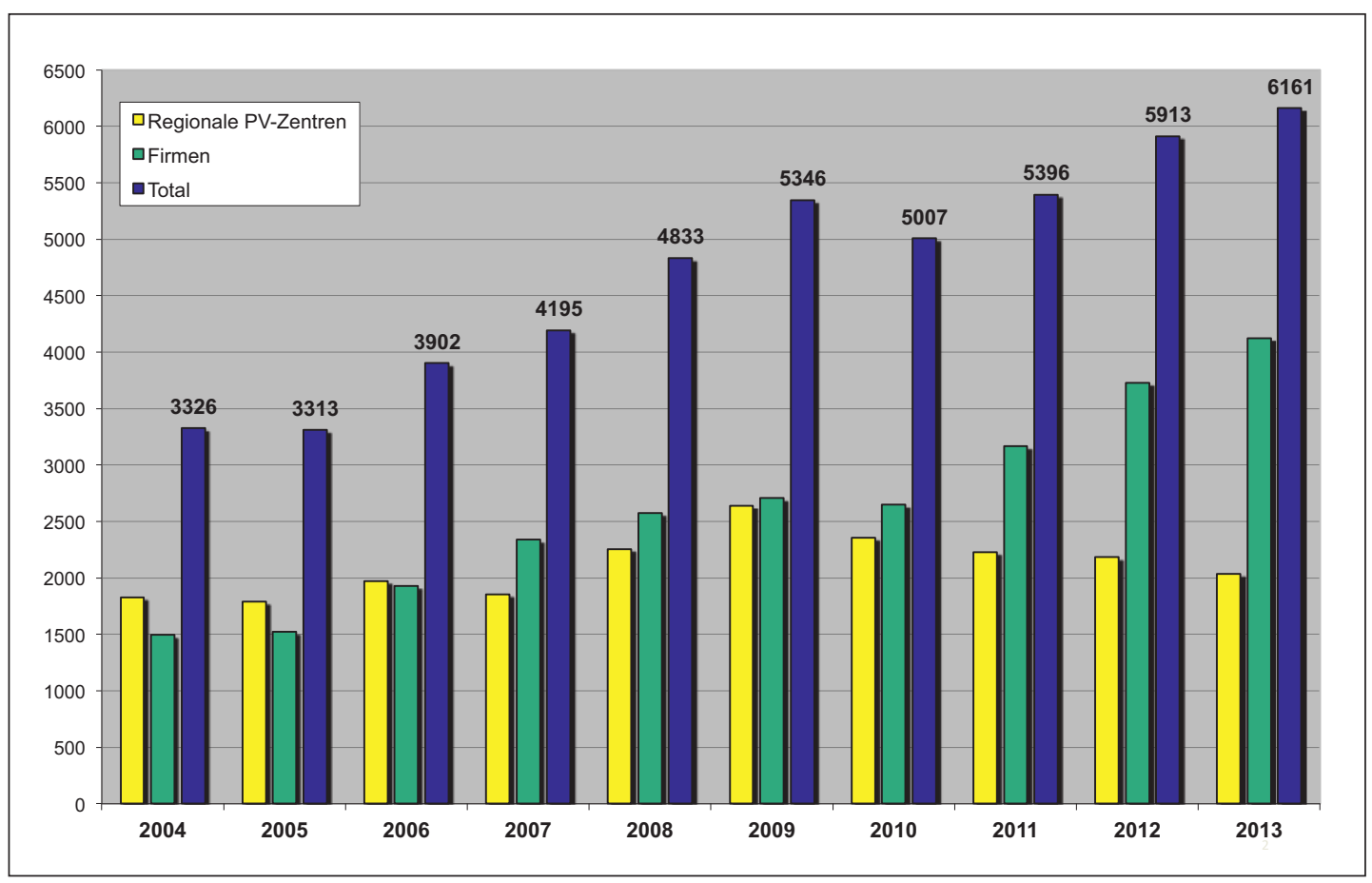

Datenbank der WHO gespeichert sowie an die betroffenen Firmen weitergeleitet.

\section{Weiterhin steigende Meldefrequenz: Anzahl Pharmacovigilance-Meldungen pro Jahr}

Die Zahl der Meldungen steigt weiterhin deutlich an (Abb. 2). Gemäss der Statistik der WHO vom März 2014 liegt die Schweiz in Europa nach Dänemark auf Rang 2, was die Zahl der UAW-Meldungen pro Million Einwohner betrifft.

Die Qualität der Berichte ist aber nicht weniger wichtig. Wir danken den meldenden Ärztinnen, Ärzten und Apotheker/innen, dass sie dazu beitragen, dass ElViS, das elektronische Vigilance-System, ab Oktober 2014 die richtigen Meldungen zur richtigen Zeit und in der richtigen Qualität transportieren kann.

\section{Referenzen}

1 Levy G, Caduff P, Stoller R. Pharmacovigilance und Spontanmeldungen unerwünschter Arzneimittelwirkungen 10 Jahre nach Inkrafttreten des Heilmittelgesetzes. Schweiz Ärztezeitung. 2013;94(4):101-4.

2 HPC - Swissmedic informiert zum Risiko schwerer Blutungen in Zusammenhang mit der Einnahme von Dabigatran (Pradaxa ${ }^{\circledast}$ ): Vorsichtsmassnahmen und therapeutische Empfehlungen. 12.4.2013. 